\title{
Lightlike Hypersurfaces of Indefinite Generalized Sasakian Space Forms
}

\author{
Dae Ho Jin \\ Department of Mathematics, Dongguk University, Gyeongju 780-714, Republic of Korea \\ Correspondence should be addressed to Dae Ho Jin; jindh@dongguk.ac.kr
}

Received 19 January 2015; Accepted 16 March 2015

Academic Editor: Dimitris Fotakis

Copyright (C) 2015 Dae Ho Jin. This is an open access article distributed under the Creative Commons Attribution License, which permits unrestricted use, distribution, and reproduction in any medium, provided the original work is properly cited.

We study lightlike hypersurfaces $M$ of an indefinite generalized Sasakian space form $\bar{M}\left(f_{1}, f_{2}\right.$, $\left.f_{3}\right)$, with indefinite trans-Sasakian structure of type $(\alpha, \beta)$, subject to the condition that the structure vector field of $\bar{M}$ is tangent to $M$. First we study the general theory for lightlike hypersurfaces of indefinite trans-Sasakian manifold of type $(\alpha, \beta)$. Next we prove several characterization theorems for lightlike hypersurfaces of an indefinite generalized Sasakian space form.

\section{Introduction}

Oubiña [1] introduced the notion of indefinite trans-Sasakian manifold of type $(\alpha, \beta)$. Indefinite Sasakian, Kenmotsu, and cosymplectic manifolds are three important kinds of indefinite trans-Sasakian manifold such that

$$
\alpha=1, \quad \beta=0 ; \quad \alpha=0, \quad \beta=1 ; \quad \alpha=\beta=0,
$$

respectively. Alegre et al. [2] introduced indefinite generalized Sasakian space form $\bar{M}\left(f_{1}, f_{2}, f_{3}\right)$. Indefinite Sasakian, Kenmotsu, and cosymplectic space forms are some kinds of indefinite generalized Sasakian space form such that

$$
\begin{gathered}
f_{1}=\frac{c+3}{4}, \quad f_{2}=f_{3}=\frac{c-1}{4} ; \\
f_{1}=\frac{c-3}{4}, \quad f_{2}=f_{3}=\frac{c+1}{4} ; \\
f_{1}=f_{2}=f_{3}=\frac{c}{4},
\end{gathered}
$$

respectively, where $c$ denotes constant $J$-sectional curvatures of each of them.

Recently author has been studying the geometry of lightlike hypersurfaces $M$ of indefinite Sasakian [3], Kenmotsu [4], and cosymplectic [5] manifolds. In this paper, we study lightlike hypersurfaces $M$ of an indefinite generalized Sasakian space form $\bar{M}\left(f_{1}, f_{2}, f_{3}\right)$, with indefinite transSasakian structure of type $(\alpha, \beta)$, subject to the condition that the structure vector field of $\bar{M}$ is tangent to $M$. First we study lightlike hypersurfaces of indefinite trans-Sasakian manifold of type $(\alpha, \beta)$. Next we prove two characterization theorems for lightlike hypersurfaces of an indefinite generalized Sasakian space form such that the following hold.

(i) Let $M$ be a lightlike hypersurface of an indefinite generalized Sasakian space form $\bar{M}\left(f_{1}, f_{2}, f_{3}\right)$. Then $\alpha$ is a constant, $\beta=0$, and

$$
f_{1}-f_{2}=\alpha^{2}, \quad f_{1}-f_{3}=\alpha^{2}, \quad f_{2}=f_{3} .
$$

(ii) Let $M$ be a screen conformal lightlike hypersurface of an indefinite generalized Sasakian space form $\bar{M}\left(f_{1}, f_{2}, f_{3}\right)$. Then $f_{1}=f_{2}=f_{3}=0$.

\section{Preliminaries}

An odd-dimensional semi-Riemannian manifold $(\bar{M}, \bar{g})$ is called an indefinite trans-Sasakian manifold $[1,2]$ if there exist 
a $(1,1)$-type tensor field $J$, a vector field $\zeta$ which is called the structure vector field, and a 1 -form $\theta$ such that

$$
\begin{gathered}
J^{2} X=-X+\theta(X) \zeta, \quad \theta(\zeta)=1, \quad J \zeta=0, \quad \theta \circ J=0 \\
\bar{g}(J X, J Y)=\bar{g}(X, Y)-\epsilon \theta(X) \theta(Y), \quad \epsilon=\bar{g}(\zeta, \zeta) \\
\left(\bar{\nabla}_{X} J\right) Y=\alpha\{\bar{g}(X, Y) \zeta-\epsilon \theta(Y) X\} \\
+\beta\{\bar{g}(J X, Y) \zeta-\epsilon \theta(Y) J X\}
\end{gathered}
$$

for any vector fields $X$ and $Y$ on $\bar{M}$, where $\epsilon=1$ or -1 according to the fact that $\zeta$ is spacelike or timelike, respectively. In this case, the set $\{J, \zeta, \theta, \bar{g}\}$ is called an indefinite trans-Sasakian structure of type $(\alpha, \beta)$.

In the entire discussion of this paper, we may assume that $\zeta$ is unit spacelike; that is, $\epsilon=1$, without loss of generality. From (4) and (6), we get

$$
\bar{\nabla}_{X} \zeta=-\alpha J X+\beta(X-\theta(X) \zeta), \quad d \theta(X, Y)=g(X, J Y)
$$

Let $(M, g)$ be a lightlike hypersurface, with a screen distribution $S(T M)$, of an indefinite trans-Sasakian manifold $\bar{M}$. Denote by $F(M)$ the algebra of smooth functions on $M$ and by $\Gamma(E)$ the $F(M)$ module of smooth sections of a vector bundle $E$. Also donate by (Equation number) $)_{i}$ the $i$ th equation of several equations in (Equation number), for example, $(7)_{1}$ donates the first equation of the two equations in (7). We use same notations for any others.

We follow Duggal-Bejancu [6] for notations and structure equations used in this paper. It is well known that, for any null section $\xi$ of $T M^{\perp}$ on a coordinate neighborhood $\mathcal{U} \subset M$, there exists a unique null section $N$ of a unique vector bundle $\operatorname{tr}(T M)$ of rank 1 in $S(T M)^{\perp}$ satisfying

$$
\begin{array}{r}
\bar{g}(\xi, N)=1, \quad \bar{g}(N, N)=\bar{g}(N, X)=0, \\
\forall X \in \Gamma(S(T M)) .
\end{array}
$$

In the following, let $X, Y, Z$, and $W$ be the vector fields on $M$, unless otherwise specified. Let $\bar{\nabla}$ be the Levi-Civita connection of $\bar{M}$ and $P$ the projection morphism of TM on $S(T M)$. Then the local Gauss and Weingarten formulas are given by

$$
\begin{aligned}
\bar{\nabla}_{X} Y & =\nabla_{X} Y+B(X, Y) N, \\
\bar{\nabla}_{X} N & =-A_{N} X+\tau(X) N ; \\
\nabla_{X} P Y & =\nabla_{X}^{*} P Y+C(X, P Y) \xi, \\
\nabla_{X} \xi & =-A_{\xi}^{*} X-\tau(X) \xi,
\end{aligned}
$$

where $\nabla$ and $\nabla^{*}$ are the liner connections on $M$ and $S(T M)$, respectively, $B$ and $C$ are the local second fundamental forms on $M$ and $S(T M)$ respectively, $A_{N}$ and $A_{\xi}^{*}$ are the shape operators on $M$ and $S(T M)$, respectively, and $\tau$ is a 1-form on $T M$.
Since $\bar{\nabla}$ is torsion-free, $\nabla$ is also torsion-free and $B$ is symmetric. From the fact that $B(X, Y)=\bar{g}\left(\bar{\nabla}_{X} Y, \xi\right)$, we show that $B$ is independent of the choice of $S(T M)$ and satisfies

$$
B(X, \xi)=0
$$

The induced connection $\nabla$ of $M$ is not metric and satisfies

$$
\left(\nabla_{X} g\right)(Y, Z)=B(X, Y) \eta(Z)+B(X, Z) \eta(Y)
$$

where $\eta$ is a 1 -form such that

$$
\eta(X)=\bar{g}(X, N)
$$

But the connection $\nabla^{*}$ on $S(T M)$ is metric. The above two local second fundamental forms $B$ and $C$ are related to their shape operators by

$$
\begin{array}{cc}
B(X, Y)=g\left(A_{\xi}^{*} X, Y\right), & \bar{g}\left(A_{\xi}^{*} X, N\right)=0, \\
C(X, P Y)=g\left(A_{N} X, P Y\right), & \bar{g}\left(A_{N} X, N\right)=0 .
\end{array}
$$

Definition 1. A lightlike hypersurface $M$ of $\bar{M}$ is said to be

(1) totally umbilical [6] if there is a smooth function $\rho$ on any coordinate neighborhood $\mathcal{U}$ in $M$ such that $A_{\xi}^{*} X=\rho P X$, or equivalently,

$$
B(X, Y)=\rho g(X, Y)
$$

In case $\rho=0$ on $\mathcal{U}$, we say that $M$ is totally geodesic;

(2) screen totally umbilical [6] if there exists a smooth function $\gamma$ on $\mathcal{U}$ such that $A_{N} X=\gamma P X$, or equivalently,

$$
C(X, P Y)=\gamma g(X, Y)
$$

In case $\gamma=0$ on $\mathcal{U}$, we say that $M$ is screen totally geodesic;

(3) screen conformal [7] if there exists a nonvanishing smooth function $\varphi$ on $\mathcal{U}$ such that $A_{N}=\varphi A_{\xi}^{*}$, or equivalently,

$$
C(X, P Y)=\varphi B(X, Y) \text {. }
$$

Denote by $\bar{R}, R$, and $R^{*}$ the curvature tensors of the LeviCivita connection $\bar{\nabla}$ of $\bar{M}$, the induced connection $\nabla$ on $M$, and the induced connection $\nabla^{*}$ on $S(T M)$, respectively. Using 
the Gauss-Weingarten formulas for $M$ and $S(T M)$, we obtain the Gauss-Codazzi equations for $M$ and $S(T M)$ such that

$$
\begin{aligned}
\bar{R}(X, Y) Z \\
=R(X, Y) Z+B(X, Z) A_{N} Y-B(Y, Z) A_{N} X \\
+\left\{\left(\nabla_{X} B\right)(Y, Z)-\left(\nabla_{Y} B\right)(X, Z)\right. \\
\quad+\tau(X) B(Y, Z)-\tau(Y) B(X, Z)\} N \\
\bar{R}(X, Y) N \\
=-\nabla_{X}\left(A_{N} Y\right)+\nabla_{Y}\left(A_{N} X\right)+A_{N}[X, Y] \\
+\tau(X) A_{N} Y-\tau(Y) A_{N} X \\
+\left\{B\left(Y, A_{N} X\right)-B\left(X, A_{N} Y\right)+2 d \tau(X, Y)\right\} N
\end{aligned}
$$

$R(X, Y) P Z$

$$
\begin{aligned}
= & R^{*}(X, Y) P Z+C(X, P Z) A_{\xi}^{*} Y \\
& -C(Y, P Z) A_{\xi}^{*} X \\
+ & \left\{\left(\nabla_{X} C\right)(Y, P Z)-\left(\nabla_{Y} C\right)(X, P Z)\right. \\
& -\tau(X) C(Y, P Z)+\tau(Y) C(X, P Z) \tau(Y)\} \xi,
\end{aligned}
$$

$R(X, Y) \xi$

$$
\begin{aligned}
= & -\nabla_{X}^{*}\left(A_{\xi}^{*} Y\right)+\nabla_{Y}^{*}\left(A_{\xi}^{*} X\right)+A_{\xi}^{*}[X, Y] \\
& -\tau(X) A_{\xi}^{*} Y+\tau(Y) A_{\xi}^{*} X \\
& +\left\{C\left(Y, A_{\xi}^{*} X\right)-C\left(X, A_{\xi}^{*} Y\right)-2 d \tau(X, Y)\right\} \xi .
\end{aligned}
$$

\section{Indefinite Trans-Sasakian Manifolds}

Let $M$ be a lightlike hypersurface of a indefinite transSasakian manifold $\bar{M}$ such that $\zeta$ is tangent to $M$. Călin [8] proved that if $\zeta$ is tangent to $M$, then it belongs to $S(T M)$ which we assume in this paper. It is well known $[3,6]$ that, for any lightlike hypersurface $M$ of an indefinite almost contact metric manifold $\bar{M}, J\left(T M^{\perp}\right)$ and $J(\operatorname{tr}(T M))$ are subbundles of $S(T M)$, of rank 1 , and $J\left(T M^{\perp}\right) \cap J(\operatorname{tr}(T M))=\{0\}$. Thus $J\left(T M^{\perp}\right) \oplus J(\operatorname{tr}(T M))$ is a subbundle of $S(T M)$ of rank 2 . First, we prove the following results.

Theorem 2. (1) Let $M$ be a totally umbilical lightlike hypersurface of an indefinite trans-Sasakian manifold $\bar{M}$. Then $\alpha=0$ and $M$ is totally geodesic.

(2) Let $M$ be a screen conformal or screen totally umbilical lightlike hypersurface of an indefinite trans-Sasakian manifold $\bar{M}$. Then $\alpha=\beta=0$. In case $M$ is screen totally umbilical, $M$ is totally geodesic.
Proof. Applying $\bar{\nabla}_{X}$ to $g(\zeta, \xi)=0$ and $g(\zeta, N)=0$, we have

$$
B(X, \zeta)=\alpha g(X, J \xi), \quad C(X, \zeta)=\alpha g(X, J N)+\beta \eta(X) .
$$

(1) If $M$ is totally umbilical, then, from (18) and (25), we have

$$
\rho g(X, \zeta)=\alpha g(X, J \xi), \quad \forall X \in \Gamma(T M)
$$

Taking $X=\zeta$ and $X=J N$ by turns, we have $\rho=0$ and $\alpha=0$, respectively. As $\rho=0, M$ is totally geodesic.

(2) If $M$ is screen conformal, then, from (20) and (25) $)_{1,2}$, we have

$$
\alpha \varphi g(X, J \xi)=\alpha g(X, J N)+\beta \eta(X)
$$

Taking $X=J \xi$ and $X=\xi$ by turns, we have $\alpha=0$ and $\beta=0$, respectively.

If $M$ is screen totally umbilical, then, from (19) and (25) , we have

$$
\gamma g(X, \zeta)=\alpha g(X, J N)+\beta \eta(X) .
$$

Taking $X=\zeta, X=J \xi$ and $X=\xi$ to this equation by turns, we have $\gamma=0, \alpha=0$, and $\beta=0$, respectively. As $\gamma=0, M$ is screen totally geodesic.

As $J\left(T M^{\perp}\right) \oplus J(\operatorname{tr}(T M))$ is a subbundle of $S(T M)$ of rank 2 , there exists a nondegenerate almost complex distribution $D_{o}$ with respect to $J$; that is, $J\left(D_{o}\right)=D_{o}$, such that

$$
\begin{aligned}
& S(T M)=\left\{J\left(T M^{\perp}\right) \oplus J(\operatorname{tr}(T M))\right\} \oplus_{\text {orth }} D_{o}, \\
& T M=\left\{J\left(T M^{\perp}\right) \oplus J(\operatorname{tr}(T M))\right\} \oplus_{\text {orth }} D_{o} \oplus_{\text {orth }} T M^{\perp} .
\end{aligned}
$$

Consider the 2-lightlike almost complex distribution $D$ such that

$$
\begin{aligned}
& D=T M^{\perp} \oplus_{\text {orth }} J\left(T M^{\perp}\right) \oplus_{\text {orth }} D_{o}, \\
& T M=D \oplus J(\operatorname{tr}(T M))
\end{aligned}
$$

and the local lightlike vector fields $U$ and $V$ and their 1-forms such that

$$
\begin{gathered}
U=-J N, \quad V=-J \xi, \quad u(X)=g(X, V), \\
v(X)=g(X, U) .
\end{gathered}
$$

Denote by $S$ the projection morphism of $T M$ on $D$. Any vector field $X$ of $M$ is expressed as $X=S X+u(X) U$. Applying $J$ to this, we have

$$
J X=F X+u(X) N,
$$

where $F$ is a tensor field of type $(1,1)$ globally defined on $M$ by

$$
F X=J S X
$$


Applying $\bar{\nabla}_{X}$ to the first two equations of (31) and (32) and using (9), (10), (12), (13), (6), (31), and (32), for any $X, Y \in$ $\Gamma(T M)$, we have

$$
\begin{gathered}
B(X, U)=C(X, V) \\
\nabla_{X} U=F\left(A_{N} X\right)+\tau(X) U-\{\alpha \eta(X)+\beta v(X)\} \zeta \\
\nabla_{X} V=F\left(A_{\xi}^{*} X\right)-\tau(X) V-\beta u(X) \zeta \\
\left(\nabla_{X} F\right)(Y)=u(Y) A_{N} X-B(X, Y) U \\
+\alpha\{g(X, Y) \zeta-\theta(Y) X\} \\
+\beta\{\bar{g}(J X, Y) \zeta-\theta(Y) F X\}
\end{gathered}
$$

Theorem 3. Let $M$ be a lightlike hypersurface of an indefinite trans-Sasakian manifold $\bar{M}$. If $V$ or $U$ is parallel with respect to $\nabla$, then $\alpha=\beta=0$ and $\tau=0$. If both $V$ and $U$ are parallel with respect to the induced connection $\nabla$, then $M$ is screen totally geodesic.

Proof. (1) If $U$ is parallel, then, from (32) and (35) we have

$$
\begin{aligned}
& J\left(A_{N} X\right)-u\left(A_{N} X\right) N+\tau(X) U-\{\alpha \eta(X)+\beta v(X)\} \zeta \\
& \quad=0 .
\end{aligned}
$$

Taking the scalar product with $V$ and $\zeta$ to (38) by turns and using (4), we have $\tau=0$ and $\alpha \eta(X)+\beta v(X)=0$, respectively. Taking $X=\xi$ and $X=V$ to the second result by turns, we have $\alpha=0$ and $\beta=0$, respectively.

(2) If $V$ is parallel with respect to $\nabla$, then, from (32) and (36), we have

$$
J\left(A_{\xi}^{*} X\right)-u\left(A_{\xi}^{*} X\right) N-\tau(X) V-\beta u(X) \zeta=0 .
$$

Taking the scalar product with $U$ to (39) and using (4), we have $\tau=0$. Taking the scalar product with $\zeta$ to (39) and using (4) and $\theta(N)=g(\zeta, N)=0$, we get $\beta u(X)=0$. Taking $X=U$ to this result, we have $\beta=0$. From $(25)_{1}$ and $(31)_{3}$, we obtain

$$
B(X, \zeta)=-\alpha u(X) .
$$

Applying $J$ to (39) and using (4) and the fact $\tau=\beta=0$, we have

$$
A_{\xi}^{*} X=\theta\left(A_{\xi}^{*} X\right) \zeta+u\left(A_{\xi}^{*} X\right) U
$$

Taking the scalar product with $U$ to this equation, we get

$$
B(X, U)=g\left(A_{\xi}^{*} X, U\right)=v\left(A_{\xi}^{*} X\right)=0 .
$$

Replacing $X$ by $U$ in (40) and using (42), we get

$$
-\alpha=-\alpha u(U)=B(U, \zeta)=0 .
$$

Thus $\alpha=\beta=0$. Then we have

$$
A_{\xi}^{*} X=u\left(A_{\xi}^{*} X\right) U
$$

(3) In case $V$ and $U$ are parallel with respect to $\nabla$, as $U$ is parallel, applying $J$ to (38) and using (4), (25) 2 and the fact $\tau=\alpha=\beta=0$, we obtain

$$
A_{N} X=u\left(A_{N} X\right) U, \quad \forall X \in \Gamma(T M) .
$$

As $V$ is parallel, from (34) and (42), we show that $u\left(A_{N} X\right)=$ $v\left(A_{\xi}^{*} X\right)=0$. Thus we obtain $A_{N}=0$. Consequently $M$ is screen totally geodesic.

Theorem 4. Let $M$ be a lightlike hypersurface of an indefinite trans-Sasakian manifold $\bar{M}$. If $F$ is parallel with respect to the connection $\nabla$, then we have $\alpha=\beta=0$. Furthermore $D$ and $J(\operatorname{tr}(T M))$ are parallel distributions on $M$ and $M$ is locally a product manifold $\mathscr{C}_{u} \times M^{\#}$, where $\mathscr{C}_{u}$ is a null curve tangent to $J(\operatorname{tr}(T M))$ and $M^{\#}$ is a leaf of $D$.

Proof. If $F$ is parallel with respect to $\nabla$, then, taking the scalar product with $U$ to $(37)$ and using the facts $g(\zeta, U)=0$ and $g(F X, U)=-\eta(X)$, we get

$$
u(Y) v\left(A_{N} X\right)-\theta(Y)\{\alpha v(X)-\beta \eta(X)\}=0 .
$$

Taking $Y=U$ and $Y=\zeta$ by turns, we get $v\left(A_{N} X\right)=0$ and $\alpha v(X)-\beta \eta(X)=0$. Taking $X=V$ and $X=\xi$ to the second equation, we have $\alpha=\beta=0$.

From (37) we have

$$
u(Y) A_{N} X=B(X, Y) U, \quad B(X, Y)=u(Y) u\left(A_{N} X\right) .
$$

Taking $Y=V$ and $Y \in \Gamma\left(D_{o}\right)$ in $(47)_{2}$ by turns, we have $B(X, V)=0$ and $B(X, Y)=0$. These results and (13) imply that

$$
B(X, Y)=0, \quad \forall X \in \Gamma(T M), Y \in \Gamma(D) .
$$

By using (4), (9), (12), (14), (32), and (36), we derive

$$
\begin{gathered}
g\left(\nabla_{X} \xi, V\right)=-g\left(\xi, \bar{\nabla}_{X} V\right)=B(X, V)=0, \\
g\left(\nabla_{X} V, V\right)=0, \\
g\left(\nabla_{X} Y, V\right)=-g\left(Y, \nabla_{X} V\right)=g\left(A_{\xi}^{*} X, J Y\right)=B(X, F Y)=0,
\end{gathered}
$$

for all $X \in \Gamma(T M)$ and $Y \in \Gamma\left(D_{o}\right)$, or equivalently, we get

$$
\nabla_{X} Y \in \Gamma(D), \quad \forall X \in \Gamma(T M), \forall Y \in \Gamma(D) .
$$

This result implies that $D$ is a parallel distribution on $M$.

Taking the scalar product with $Z \in \Gamma\left(D_{o}\right)$ to $(47)_{1}$, we get $u(Y) C(X, Z)=0$ for all $X, Y \in \Gamma(T M)$. Taking $Y=U$ to this, we have

$$
C(X, Y)=0, \quad \forall X \in \Gamma(T M), Y \in \Gamma\left(D_{o}\right) .
$$

For all $X \in \Gamma(T M)$ and $Y \in \Gamma\left(D_{o}\right)$, using (35) we derive

$$
\begin{aligned}
g\left(\nabla_{X} U, N\right) & =v\left(A_{N} X\right)=0, \\
g\left(\nabla_{X} U, U\right) & =-g\left(A_{N} X, N\right)=0, \\
g\left(\nabla_{X} U, Y\right) & =g\left(F\left(A_{N} X\right), Y\right) \\
& =-g\left(A_{N} X, J Y\right)=C(X, F Y)=0 ;
\end{aligned}
$$


that is, $\nabla_{X} U \in \Gamma(J(\operatorname{tr}(T M)))$ for all $X \in \Gamma(T M)$. Thus $J(\operatorname{tr}(T M))$ is also parallel. As $T M=D \oplus J(\operatorname{tr}(T M))$, and $D$ and $J(\operatorname{tr}(T M))$ are parallel distributions, by the decomposition theorem of de Rham [9] we have $M=\mathscr{C}_{u} \times M^{\#}$, where $\mathscr{C}_{u}$ is a null curve tangent to $J(\operatorname{tr}(T M))$ and $M^{*}$ is a leaf of $D$.

Corollary 5. Let $M$ be a lightlike hypersurface of an indefinite trans-Sasakian manifold $\bar{M}$. If $F$ and $V$ are parallel with respect to $\nabla$, then $M$ is totally geodesic and screen totally geodesic.

Proof. As $F$ is parallel with respect to $\nabla$, we get the two equations of (47). As $V$ is also parallel with respect to $\nabla$, substituting (34) to (47) $)_{2}$ and using (42), we have $B=0$. Thus $M$ is totally geodesic. Replacing $Y$ by $U$ to $(47)_{1}$, we obtain $A_{N}=0$. Thus $M$ is also screen totally geodesic.

\section{Indefinite Generalized Sasakian Space Form}

An indefinite almost contact metric manifold $(\bar{M}, J, \zeta, \theta, \bar{g})$ is said to be an indefinite generalized Sasakian space form [2] and denote it by $\bar{M}\left(f_{1}, f_{2}, f_{3}\right)$ if there exist three smooth functions $f_{1}, f_{2}$, and $f_{3}$ on $\bar{M}$ such that

$$
\begin{aligned}
\bar{R}(X, Y) Z & \\
= & f_{1}\{\bar{g}(Y, Z) X-\bar{g}(X, Z) Y\} \\
& +f_{2}\{\bar{g}(X, J Z) J Y-\bar{g}(Y, J Z) J X+2 \bar{g}(X, J Y) J Z\} \\
& +f_{3}\{\theta(X) \theta(Z) Y-\theta(Y) \theta(Z) X \\
& \quad+\bar{g}(X, Z) \theta(Y) \zeta-\bar{g}(Y, Z) \theta(X) \zeta\},
\end{aligned}
$$

for any vector fields $X, Y$, and $Z$ on $\bar{M}$.

Theorem 6. Let $M$ be a lightlike hypersurface of an indefinite generalized Sasakian space form $\bar{M}\left(f_{1}, f_{2}, f_{3}\right)$. Then $\alpha$ is a constant, $\beta=0$, and

$$
f_{1}-f_{2}=\alpha^{2}, \quad f_{1}-f_{3}=\alpha^{2}, \quad f_{2}=f_{3} .
$$

Proof. Comparing the tangential and transversal components of (21) and (53), and using (32), we get

$$
\begin{aligned}
R(X, Y) Z \\
=f_{1}\{g(Y, Z) X-g(X, Z) Y\} \\
+f_{2}\{\bar{g}(X, J Z) F Y-\bar{g}(Y, J Z) F X+2 \bar{g}(X, J Y) F Z\} \\
+f_{3}\{\theta(X) \theta(Z) Y-\theta(Y) \theta(Z) X \\
\quad+\bar{g}(X, Z) \theta(Y) \zeta-\bar{g}(Y, Z) \theta(X) \zeta\} \\
+B(Y, Z) A_{N} X-B(X, Z) A_{N} Y,
\end{aligned}
$$

$$
\begin{aligned}
&\left(\nabla_{X} B\right)(Y, Z)-\left(\nabla_{Y} B\right)(X, Z) \\
&+ \tau(X) B(Y, Z)-\tau(Y) B(X, Z) \\
&=f_{2}\{u(Y) \bar{g}(X, J Z)-u(X) \bar{g}(Y, J Z) \\
&+2 u(Z) \bar{g}(X, J Y)\} .
\end{aligned}
$$

Taking the scalar product with $N$ to (23), we have

$$
\begin{aligned}
g(R(X, Y) P Z, N)= & \left(\nabla_{X} C\right)(Y, P Z)-\left(\nabla_{Y} C\right)(X, P Z) \\
& -\tau(X) C(Y, P Z)+\tau(Y) C(X, P Z) .
\end{aligned}
$$

Substituting (55) into the last equation and using $(17)_{2}$, we obtain

$$
\begin{aligned}
\left(\nabla_{X} C\right) & (Y, P Z)-\left(\nabla_{Y} C\right)(X, P Z) \\
- & \tau(X) C(Y, P Z)+\tau(Y) C(X, P Z) \\
= & f_{1}\{g(Y, P Z) \eta(X)-g(X, P Z) \eta(Y)\} \\
+ & f_{2}\{v(Y) \bar{g}(X, J P Z)-v(X) \bar{g}(Y, J P Z) \\
& +2 v(P Z) \bar{g}(X, J Y)\} \\
+ & f_{3}\{\theta(X) \eta(Y)-\theta(Y) \eta(X)\} \theta(P Z) .
\end{aligned}
$$

Applying $\nabla_{X}$ to $(34)_{1}: B(Y, U)=C(Y, V)$, we have

$$
\begin{aligned}
& \left(\nabla_{X} B\right)(Y, U) \\
& \quad=\left(\nabla_{X} C\right)(Y, V)+g\left(A_{N} Y, \nabla_{X} V\right)-g\left(A_{\xi}^{*} Y, \nabla_{X} U\right) .
\end{aligned}
$$

Using (25), (32), (34), (35), and (36), the above equation is reduced to

$$
\begin{aligned}
\left(\nabla_{X} B\right)(Y, U)= & \left(\nabla_{X} C\right)(Y, V)-2 \tau(X) C(Y, V) \\
& -\alpha^{2} u(Y) \eta(X)-\beta^{2} u(X) \eta(Y) \\
& +\alpha \beta\{u(X) v(Y)-u(Y) v(X)\} \\
& -g\left(A_{\xi}^{*} X, F\left(A_{N} Y\right)\right)-g\left(A_{\xi}^{*} Y, F\left(A_{N} X\right)\right) .
\end{aligned}
$$

Substituting this equation and (34) into (56) such that $Z=U$, we get

$$
\begin{aligned}
\left(\nabla_{X} C\right) & (Y, V)-\left(\nabla_{Y} C\right)(X, V)-\tau(X) C(Y, V) \\
& +\tau(Y) C(X, V)+\left(\alpha^{2}-\beta^{2}\right)\{u(X) \eta(Y)-u(Y) \eta(X)\} \\
& +2 \alpha \beta\{u(X) v(Y)-u(Y) v(X)\} \\
= & f_{2}\{u(Y) \eta(X)-u(X) \eta(Y)+2 \bar{g}(X, J Y)\} .
\end{aligned}
$$

Comparing this equation with (58) such that $P Z=V$, we obtain

$$
\begin{aligned}
& \left\{f_{1}-f_{2}-\left(\alpha^{2}-\beta^{2}\right)\right\}[u(Y) \eta(X)-u(X) \eta(Y)] \\
& \quad=2 \alpha \beta\{u(Y) v(X)-u(X) v(Y)\} .
\end{aligned}
$$


Taking $X=\xi$ and $Y=U$ and $X=V$ and $Y=U$ by turns, we have

$$
f_{1}-f_{2}=\alpha^{2}-\beta^{2}, \quad \alpha \beta=0 .
$$

Substituting (32) into (7) and using (9), we have

$$
\nabla_{X} \zeta=-\alpha F X+\beta(X-\theta(X) \zeta), \quad \forall X \in \Gamma(T M)
$$

Applying $\bar{\nabla}_{X}$ to $v(Y)=g(Y, U)$ and using (9), (32), (34), and (35), we get

$$
\begin{aligned}
\left(\nabla_{X} v\right)(Y)= & v(Y) \tau(X)-\theta(Y)\{\alpha \eta(X)+\beta v(X)\} \\
& -g\left(A_{N} X, F Y\right)
\end{aligned}
$$

Applying $\bar{\nabla}_{X}$ to $\eta(Y)=\bar{g}(Y, N)$ and using (4) and (6) we have

$$
\left(\nabla_{X} \eta\right)(Y)=-g\left(A_{N} X, Y\right)+\tau(X) \eta(Y) .
$$

Using (31), the equation $(25)_{2}$ is reduced to

$$
C(Y, \zeta)=-\alpha v(Y)+\beta \eta(Y) \text {. }
$$

Applying $\nabla_{X}$ to this equation and using (64), (65), and (66), we have

$$
\begin{aligned}
\left(\nabla_{X} C\right) & (Y, \zeta) \\
= & -(X \alpha) v(Y)+(X \beta) \eta(Y)-\alpha \tau(X) v(Y) \\
& +\alpha^{2} \theta(Y) \eta(X)+\beta^{2} \theta(X) \eta(Y) \\
& -\beta\left\{g\left(X, A_{N} Y\right)+g\left(A_{N} X, Y\right)-\tau(X) \eta(Y)\right\} \\
& +\alpha\left\{g\left(A_{N} X, F Y\right)+g\left(A_{N} Y, F X\right)\right\} .
\end{aligned}
$$

Substituting this and (67) into (58) such that $P Z=\zeta$, we get

$$
\begin{aligned}
& \{X \beta+A \theta(X)\} \eta(Y)-\{Y \beta+A \theta(Y)\} \eta(X) \\
& =(X \alpha) v(Y)-(Y \alpha) v(X),
\end{aligned}
$$

where $A=f_{1}-f_{3}-\left(\alpha^{2}-\beta^{2}\right)$. Taking $X=\xi$ and $Y=\zeta$ and then taking $X=U$ and $Y=V$ to this equation, we obtain

$$
f_{1}-f_{3}=\left(\alpha^{2}-\beta^{2}\right)-\zeta \beta, \quad U \alpha=0 .
$$

Applying $\bar{\nabla}_{X}$ to $u(Y)=g(Y, V)$ and using (9), (32), and (36), we get

$$
\left(\nabla_{X} u\right)(Y)=-u(Y) \tau(X)-\beta \theta(Y) u(X)-B(X, F Y) .
$$

Applying $\nabla_{Y}$ to (40) and using (40) and (64) and (71), we have

$$
\begin{aligned}
\left(\nabla_{X} B\right)(Y, \zeta)= & -(X \alpha) u(Y)-\beta B(X, Y) \\
& +\alpha\{u(Y) \tau(X)+B(X, F Y)+B(Y, F X)\} .
\end{aligned}
$$

Substituting this into (56) such that $Z=\zeta$ and using the fact that $U \alpha=0$, we have $(X \alpha) u(Y)=0$. Therefore the function $\alpha$ is a constant.
From the facts that $\alpha$ is a constant and $\alpha \beta=0$, if $\alpha \neq 0$, then we get $\beta=0$.

Assume that $\alpha=0$. Then (64) is reduced to

$$
\nabla_{Y} \zeta=\beta(Y-\theta(Y) \zeta)
$$

By straightforward calculations form this equation, we obtain

$$
\begin{aligned}
R(X, Y) \zeta= & (X \beta) Y-(Y \beta) X-\{(X \beta) \theta(Y)-(Y \beta) \theta(X)\} \zeta \\
& +\beta^{2}\{\theta(X) Y-\theta(Y) X\}-2 \beta d \theta(X, Y) \zeta
\end{aligned}
$$

Comparing this equation with (55) such that $Z=\zeta$, we obtain

$$
\begin{aligned}
(X \beta) & Y-(Y \beta) X-\{(X \beta) \theta(Y)-(Y \beta) \theta(X)\} \zeta \\
+ & \beta^{2}\{\theta(X) Y-\theta(Y) X\}-2 \beta d \theta(X, Y) \zeta \\
= & \left(f_{1}-f_{3}\right)\{\theta(Y) X-\theta(X) Y\} .
\end{aligned}
$$

Taking the scalar product with $\zeta$ to this equation, we get $\beta d \theta(X, Y)=0$; that is,

$$
\beta g(X, J Y)=0, \quad \forall X, Y \in \Gamma(T M),
$$

due to $(32)_{2}$. Taking $X=U$ and $Y=\xi$ to this equation, we have $\beta=0$.

As $\beta=0,(63)$ and (70) are reduced to $f_{1}-f_{2}=\alpha^{2}$ and $f_{1}-f_{3}=\alpha^{2}$, respectively. From these two results, we get $f_{2}=$ $f_{3}$.

Corollary 7. There exist no indefinite generalized Sasakian space forms, endowed with $\beta$-Kenmotsu structure, admitting a lightlike hypersurface.

Corollary 8. Let $M$ be a lightlike hypersurface of an indefinite Sasakian space form $\bar{M}(c)$, endowed with $\alpha$-Sasakian structure. Then $\alpha= \pm 1$.

Theorem 9. Let $M$ is lightlike hypersurface of an indefinite generalized Sasakian space form $\bar{M}\left(f_{1}, f_{2}, f_{3}\right)$. If $M$ is screen totally umbilical, then $f_{1}=f_{2}=f_{3}=0$.

Proof. As $M$ is screen totally umbilical, $\alpha=\beta=C=0$ by (2) of Theorem 2. Thus (58) is reduced to

$$
\begin{aligned}
& f_{1}\{g(Y, P Z) \eta(X)-g(X, P Z) \eta(Y)\} \\
& +f_{2}\{v(Y) \bar{g}(X, J P Z)-v(X) \bar{g}(Y, J P Z) \\
& \quad+2 v(P Z) \bar{g}(X, J Y)\} \\
& +f_{3}\{\theta(X) \theta(P Z) \eta(Y)-\theta(Y) \theta(P Z) \eta(X)\}=0,
\end{aligned}
$$

for all $X, Y, Z \in \Gamma(T M)$. Replacing $Y$ by $\xi$ to this equation, we obtain

$$
\begin{aligned}
& f_{1} g(X, P Z)+f_{2}\{v(X) u(P Z)+2 u(X) v(P Z)\} \\
& \quad-f_{3} \theta(X) \theta(P Z)=0 .
\end{aligned}
$$


Taking $X=V, P Z=U ; X=U, P Z=V$, and $X=P Z=\zeta$ by turns, we have

$$
f_{1}+f_{2}=0, \quad f_{1}+2 f_{2}=0, \quad f_{1}=f_{3} .
$$

From the first two equations we show that $f_{2}=0$. As $\alpha=\beta=$ $0, \bar{M}$ is an indefinite cosymplectic manifold. Thus $f_{1}=f_{2}=$ $f_{3}=c / 4$. This implies $f_{1}=f_{2}=f_{3}=0$.

Theorem 10. Let $M$ be a screen conformal lightlike hypersurface of an indefinite generalized Sasakian space form $\bar{M}\left(f_{1}, f_{2}, f_{3}\right)$. Then $f_{1}=f_{2}=f_{3}=0$.

Proof. Substituting (55) into (57) and using (56), we have

$$
\begin{aligned}
f_{1}\{g(Y, P Z) \eta(X)-g(X, P Z) \eta(Y)\} \\
+f_{2}\{[v(Y)-u(Y)] \bar{g}(X, J P Z) \\
-[v(X)-u(X)] \bar{g}(Y, J P Z) \\
+2[v(P Z)-u(P Z)] \bar{g}(X, J Y)\} \\
+f_{3}\{\theta(X) \theta(P Z) \eta(Y)-\theta(Y) \theta(P Z) \eta(X)\} \\
=\{X[\varphi]-2 \varphi \tau(X)\} B(Y, P Z) \\
-\{Y[\varphi]-2 \varphi \tau(Y)\} B(X, P Z) .
\end{aligned}
$$

Replacing $Y$ by $\xi$ to the last equation, we obtain

$$
\begin{aligned}
\{\xi[\varphi]-2 \varphi \tau(\xi)\} B(X, P Z) \\
=f_{1} g(X, P Z)+f_{2}\{v(X)-u(X)\} u(P Z) \\
\quad+2 f_{2}\{v(P Z)-u(P Z)\} u(X)-f_{3} \theta(X) \theta(P Z) .
\end{aligned}
$$

Taking $X=P Z=\zeta$ to this equation and using (40), we obtain $f_{1}=f_{3}$. Also taking $X=V, P Z=U$, and $X=U, P Z=V$ by turns, we have

$$
\begin{aligned}
& \{\xi[\varphi]-2 \varphi \tau(\xi)\} B(V, U)=f_{1}+f_{2}, \\
& \{\xi[\varphi]-2 \varphi \tau(\xi)\} B(U, V)=f_{1}+2 f_{2},
\end{aligned}
$$

respectively. Comparing these two equations, we obtain $f_{2}=$ 0 .

As $M$ is screen conformal, we obtain $\alpha=\beta=0$ by Theorem 2. As $\alpha=\beta=0$, we show that $\bar{M}$ is a cosymplectic manifold and $f_{1}=f_{2}=f_{3}=c / 4$. Therefore we get $f_{1}=f_{2}=$ $f_{3}=0$. by

Let $R^{(0,2)}$ denote the induced Ricci type tensor of $M$ given

$$
R^{(0,2)}(X, Y)=\operatorname{trace}\{Z \longrightarrow R(Z, X) Y\},
$$

for any $X, Y \in \Gamma(T M)$. Consider the induced quasi-orthonormal frame field $\left\{\xi ; W_{a}\right\}$ on $M$ such that $T M^{\perp}=\operatorname{Span}\{\xi\}$ and $S(T M)=\operatorname{Span}\left\{W_{a}\right\}$. Put $m=\operatorname{rank}(S(T M))$. Using this quasi-orthonormal frame field, we obtain

$$
R^{(0,2)}(X, Y)=\sum_{a=1}^{m} \epsilon_{a} g\left(R\left(W_{a}, X\right) Y, W_{a}\right)+\bar{g}(R(\xi, X) Y, N),
$$

for any $X, Y \in \Gamma(T M)$, where $\epsilon_{a}=g\left(W_{a}, W_{a}\right)$ is the causal character of $W_{a}$. In general, the induced Ricci type tensor $R^{(0,2)}$ is not symmetric $[6,7]$. A tensor field $R^{(0,2)}$ of lightlike submanifolds $M$ is called its induced Ricci tensor if it is symmetric. A symmetric $R^{(0,2)}$ tensor will be denoted by Ric. A lightlike manifold $M$ equipped with an induced Ricci tensor is called Ricciflat if its Ricci tensor vanishes. $M$ is called an Einstein manifold if the Ricci tensor of $M$ satisfies Ric $=\gamma g$.

If $M$ is a screen conformal lightlike hypersurface of $\bar{M}\left(f_{1}, f_{2}, f_{3}\right)$, then, using (55) and the fact that $f_{1}=f_{2}=$ $f_{3}=0$, we have

$$
R^{(0,2)}(X, Y)=\varphi\left\{B(X, Y) \operatorname{tr} A_{\xi}^{*}-g\left(A_{\xi}^{*} X, A_{\xi}^{*} Y\right)\right\} .
$$

This implies that $R^{(0,2)}$ is a symmetric induced Ricci tensor Ric.

Theorem 11. Any screen conformal Einstein lightlike hypersurface of an indefinite generalized Sasakian space form $\bar{M}\left(f_{1}, f_{2}, f_{3}\right)$ is Ricci flat.

Proof. As $M$ is Einstein, from (85) and the fact $R^{(0,2)}=\gamma g$

$$
g\left(A_{\xi}^{*} X, A_{\xi}^{*} Y\right)-\alpha g\left(A_{\xi}^{*} X, Y\right)-\gamma \varphi^{-1} g(X, Y)=0,
$$

where $\alpha=\operatorname{tr} A_{\xi}^{*}$ is trace of $A_{\xi}^{*}$. Define a nonnull vector field $\mu$ on $S(T M)$ by

$$
\mu=U-\varphi V
$$

Then $\mu$ belongs to $J\left(T M^{\perp}\right) \oplus J(\operatorname{tr}(T M))$. Using (20) and (34), $\mu$ satisfies

$$
B(X, \mu)=0, \quad \forall X \in \Gamma(T M) .
$$

From this equation and (16), we show that

$$
A_{\xi}^{*} \mu=0 .
$$

Taking $X=Y=\mu$ to (86) and using (89), we get $\gamma=0$. Therefore, $M$ is Ricci flat.

\section{Parallel Structure Fields}

Definition 12. Let $\nabla_{X}^{\perp} N=\pi\left(\bar{\nabla}_{X} N\right)$ for any $X \in \Gamma(T M)$, where $\pi$ is the projection morphism of $T \bar{M}$ on $\operatorname{tr}(T M)$. Then $\nabla^{\perp}$ is a linear connection on $\operatorname{ltr}(T M)$. We say that $\nabla^{\perp}$ is the transversal connection of $M$. We define the curvature tensor $R^{\perp}$ of $\operatorname{tr}(T M)$ by

$$
R^{\perp}(X, Y) N=\nabla_{X}^{\perp} \nabla_{Y}^{\perp} N-\nabla_{Y}^{\perp} \nabla_{X}^{\perp} N-\nabla_{[X, Y]}^{\perp} N .
$$

The transversal connection of $M$ is flat [3] if $R^{\perp}$ vanishes.

As $\nabla_{X}^{\perp} N=\tau(X) N$, we show that the transversal connection of $M$ is flat if and only if the 1 -form $\tau$ is closed; that is, $d \tau=0$, on any $\mathcal{U} \subset M[3]$

Denote $\lambda$ and $\mu$ by the 1 -forms such that

$$
\lambda(X)=B(X, U)=C(X, V), \quad \delta(X)=B(X, V) .
$$


Theorem 13. Let $M$ be a lightlike hypersurface of an indefinite generalized Sasakian space form $\bar{M}\left(f_{1}, f_{2}, f_{3}\right)$. If one of the following conditions,

(1) $F$ is parallel with respect to the connection $\nabla$,

(2) $U$ is parallel with respect to the connection $\nabla$,

(3) $V$ is parallel with respect to the connection $\nabla$,

is satisfied, then $\bar{M}\left(f_{1}, f_{2}, f_{3}\right)$ is a flat manifold with indefinite cosymplectic structure and the lightlike transversal connection of $M$ is flat. In case (1), $M$ is also a flat manifold.

Proof. (1) Assume that $F$ is parallel with respect to $\nabla$. Then we get $\alpha=\beta=0$ by Theorem 4 . Thus $f_{1}=f_{2}=f_{3}$ by Theorem 6 and (37) is reduced to

$$
u(Y) A_{N} X-B(X, Y) U=0 .
$$

Taking $Y=U$ to (92) and using (31), we have

$$
A_{N} X=\lambda(X) U \text {. }
$$

Taking the scalar product with $V$ to (92) and using (17) and (31), we have

$$
g\left(A_{\xi}^{*} X, Y\right)=g(\lambda(X) V, Y) .
$$

As $A_{\xi}^{*} X$ and $V$ belong to $S(T M)$ and $S(T M)$ is nondegenerate, we have

$$
A_{\xi}^{*} X=\lambda(X) V
$$

Taking the scalar product with $U$ to (93), we obtain

$$
C(X, U)=0 .
$$

Applying $\nabla_{X}$ to $C(Y, U)=0$ and using (37), (93) and $F U=0$, we get

$$
\left(\nabla_{X} C\right)(Y, U)=0 .
$$

Replacing $P Z$ by $U$ to (58) and using the last two equations, we have

$$
f_{1}\{v(Y) \eta(X)-v(X) \eta(Y)\}=0 .
$$

Taking $X=V$ and $Y=\xi$ to this equation, we get $f_{1}=0$. Therefore, $f_{1}=f_{2}=f_{3}=0$ and $\bar{M}\left(f_{1}, f_{2}, f_{3}\right)$ is flat. we get

As $f_{1}=f_{2}=f_{3}=0$, substituting (93) and (95) into (55),

$$
\begin{aligned}
R(X, Y) Z= & \{\lambda(Y) \lambda(X)-\lambda(X) \lambda(Y)\} u(Z) U \\
& +\{\sigma(Y) \sigma(X)-\sigma(X) \sigma(Y)\} w(Z) W=0 .
\end{aligned}
$$

Thus $M$ is flat. From (37), (93) and the fact that $F U=\rho=0$, we get

$$
\nabla_{X} U=\tau(X) U
$$

Substituting this equation into $\nabla_{X} \nabla_{Y} U-\nabla_{Y} \nabla_{X} U-\nabla_{[X, Y]} U=0$, we get $d \tau=0$. Thus the transversal connection of $M$ is flat.

(2) If $U$ is parallel with respect to $\nabla$, then, $\alpha=\beta=\tau=0$ by Theorem 3. Thus $f_{1}=f_{2}=f_{3}$ by Theorem 6 and (35) is reduced to

$$
J\left(A_{N} X\right)-u\left(A_{N} X\right) N=0 .
$$

Applying $J$ to (101) and using (4), (31), and (67), we have

$$
A_{N} X=\lambda(X) U .
$$

Taking the scalar product with $U$ to (102), we get

$$
C(X, U)=0 .
$$

Applying $\nabla_{Y}$ to this and using (35), (102) and the fact that $F U=0$, we get

$$
\left(\nabla_{X} C\right)(Y, U)=0 .
$$

Substituting the last two equation into (58) such that $P Z=U$, we have

$$
f_{1}\{v(Y) \eta(X)-v(X) \eta(Y)\}=0 .
$$

Taking $X=V$ and $Y=\xi$ to this equation, we obtain $f_{1}=0$. Therefore, $f_{1}=f_{2}=f_{3}=0$ and $\bar{M}\left(f_{1}, f_{2}, f_{3}\right)$ is flat. As $\tau=0$, we obtain $d \tau=0$. Thus the transversal connection of $M$ is flat.

(3) If $V$ is parallel with respect to $\nabla$, then, $\alpha=\beta=\tau=0$ by Theorem 3. Thus $f_{1}=f_{2}=f_{3}$ by Theorem 6 and (35) is reduced to

$$
J\left(A_{\xi}^{*} X\right)-u\left(A_{\xi}^{*} X\right) N=0 .
$$

Applying $J$ to (106) and using (4) and (40), we have

$$
A_{\xi}^{*} X=\mu(X) U .
$$

Taking the scalar product with $U$ to this equation, we get

$$
B(X, U)=0 .
$$

Applying $\nabla_{Y}$ to this equation and using (35), we have

$$
\left(\nabla_{X} B\right)(Y, U)=-B\left(Y, F\left(A_{N} X\right)\right) .
$$

Substituting the last two equations into (56), we obtain

$$
\begin{aligned}
B & \left(X, F\left(A_{N} Y\right)\right)-B\left(Y, F\left(A_{N} X\right)\right) \\
& =f_{2}\{u(Y) \eta(X)-u(X) \eta(Y)+2 \bar{g}(X, J Y)\} .
\end{aligned}
$$

Taking $X=\xi$ and $Y=U$ to this equation and using (14) and (108), we obtain $f_{2}=0$. Therefore, $f_{1}=f_{2}=f_{3}=0$ and $\bar{M}\left(f_{1}, f_{2}, f_{3}\right)$ is flat. As $\tau=0$, we obtain $d \tau=0$. Thus the lightlike transversal connection of $M$ is flat.

\section{Conflict of Interests}

The author declares that there is no conflict of interests regarding the publication of this paper. 


\section{References}

[1] J. A. Oubiña, "New classes of almost contact metric structures," Publicationes Mathematicae Debrecen, vol. 32, no. 3-4, pp. 187193, 1985.

[2] P. Alegre, D. E. Blair, and A. Carriazo, "Generalized Sasakianspace-forms," Israel Journal of Mathematics, vol. 141, pp. 157-183, 2004.

[3] D. H. Jin, "Geometry of lightlike hypersurfaces of an indefinite Sasakian manifold," Indian Journal of Pure and Applied Mathematics, vol. 41, no. 4, pp. 569-581, 2010.

[4] D. H. Jin, "The curvatures of lightlike hypersurfaces of an indefinite Kenmotsu manifold," Balkan Journal of Geometry and its Applications, vol. 17, no. 1, pp. 49-57, 2012.

[5] D. H. Jin, "Geometry of lightlike hypersurfaces of an indefinite cosymplectic manifold," Communications of the Korean Mathematical Society, vol. 27, no. 1, pp. 185-195, 2012.

[6] K. L. Duggal and A. Bejancu, Lightlike Submanifolds of SemiRiemannian Manifolds and Applications, vol. 364, Kluwer Academic Publishers, Dordrecht, The Netherlands, 1996.

[7] K. L. Duggal and D. H. Jin, Null curves and Hypersurfaces of semi-Riemannian manifolds, World Scientific, River Edge, NJ, USA, 2007.

[8] C. Călin, Contributions to geometry of CR-submanifold [M.S. thesis], University of Iasi, Iasi, Romania, 1998.

[9] G. de Rham, "Sur la réductibilité d'un espace de Riemann," Commentarii Mathematici Helvetici, vol. 26, pp. 328-344, 1952. 


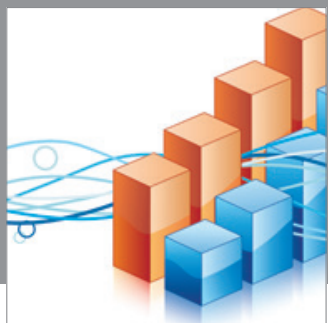

Advances in

Operations Research

mansans

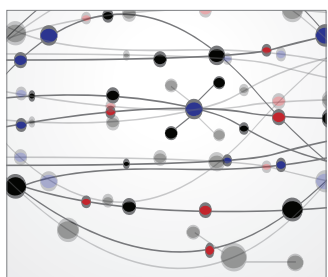

The Scientific World Journal
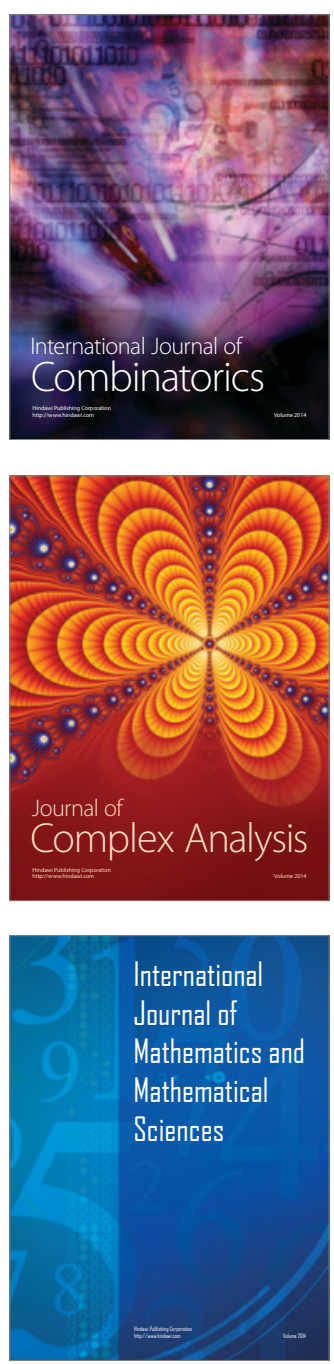
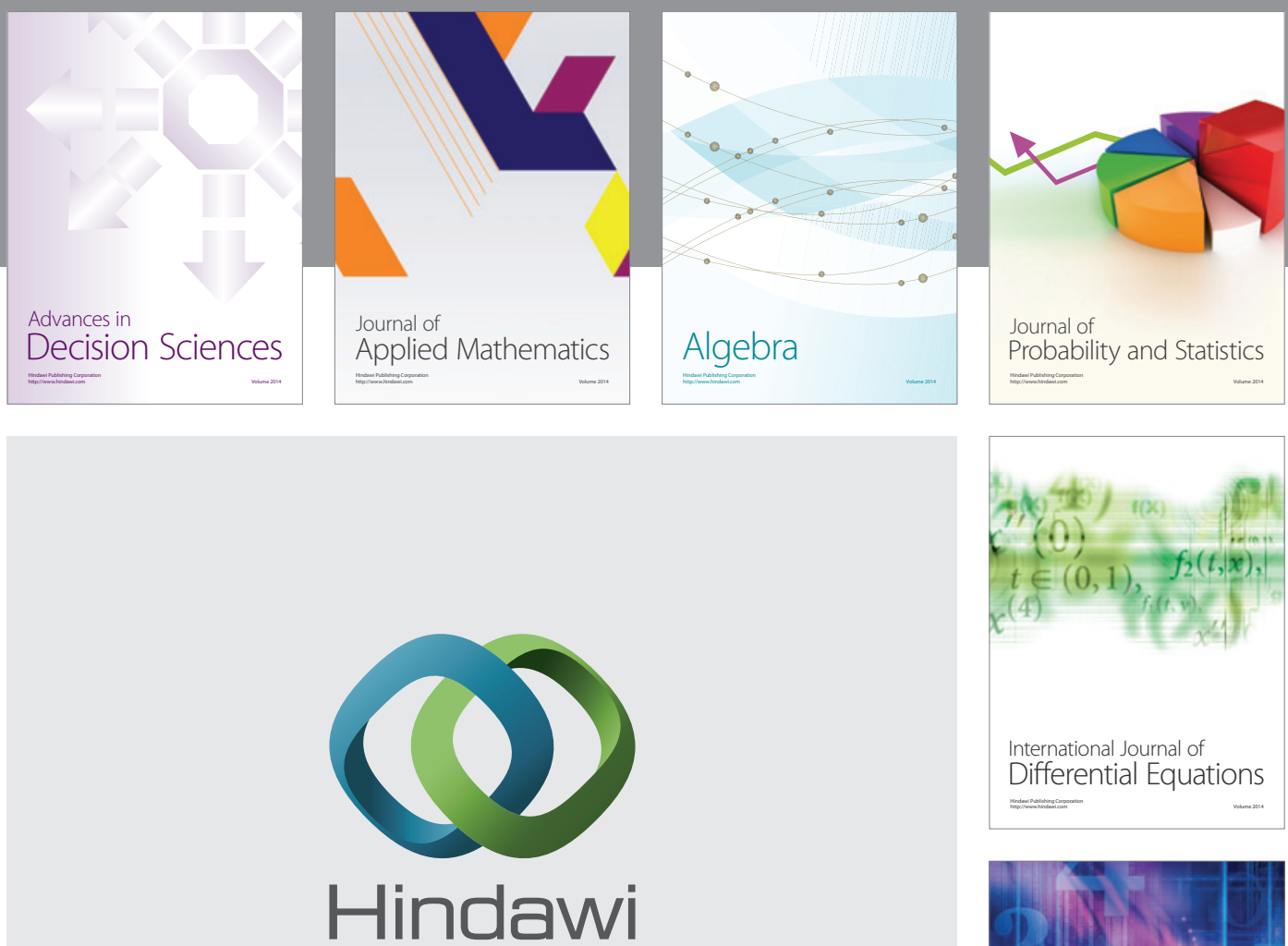

Submit your manuscripts at http://www.hindawi.com
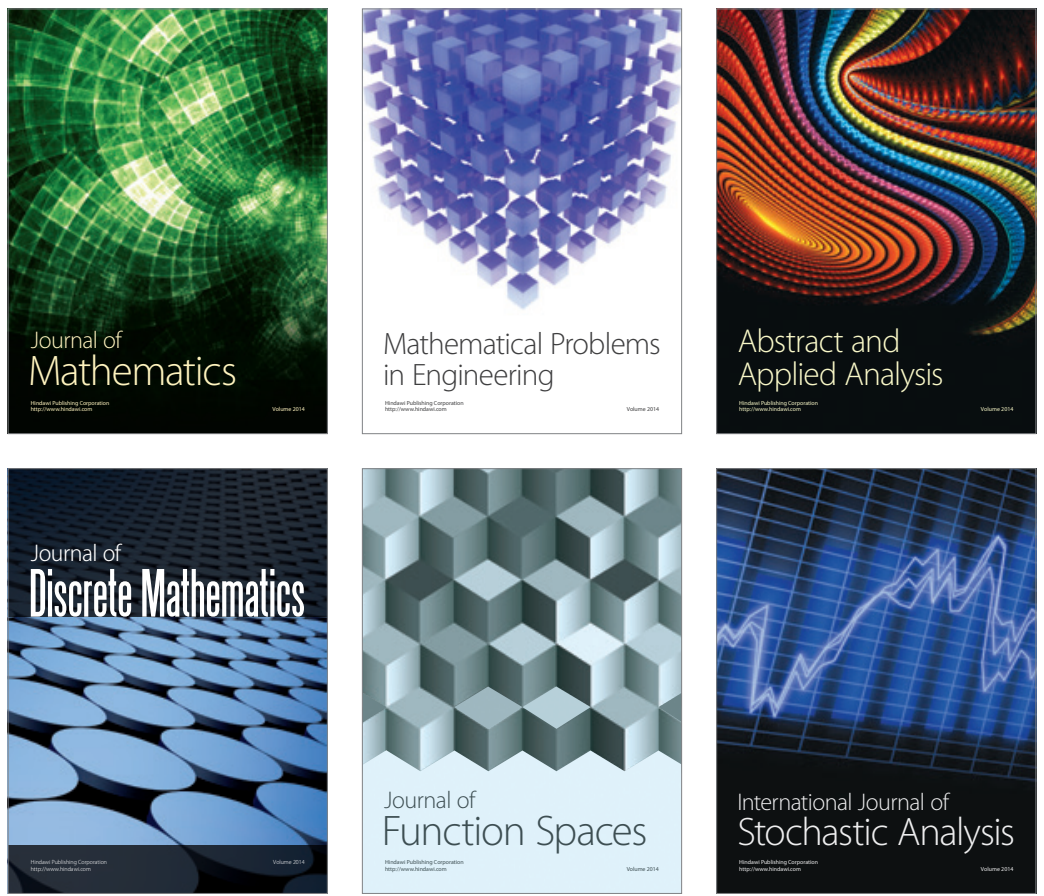

Journal of

Function Spaces

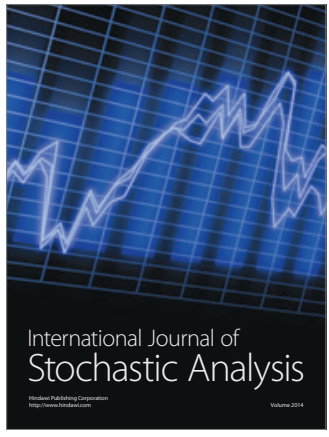

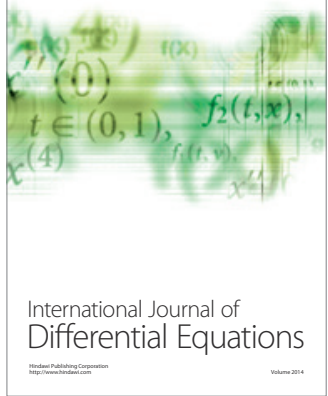
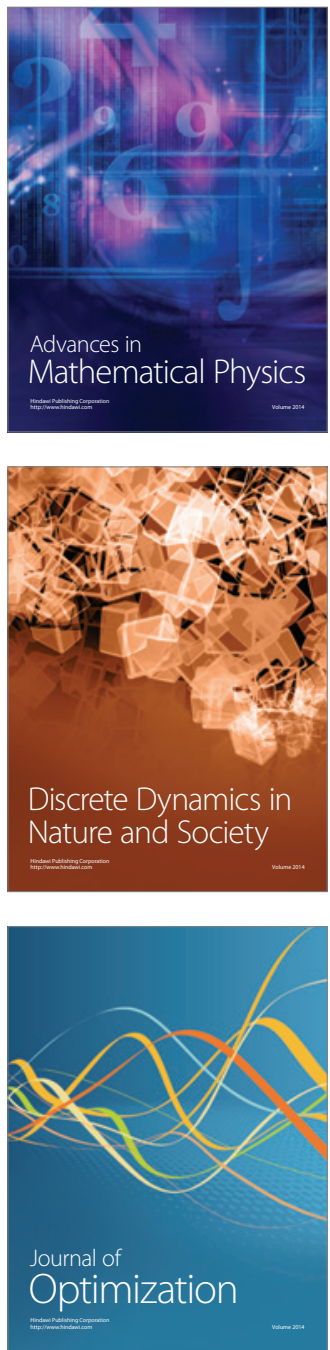